\title{
Obtención de curvas de motores de inducción usando un circuito equivalente de parámetros variables
}

\section{Obtaining induction motor curves using an equivalent circuit with variable parameters}

Presentación: 07/08/2021

Aprobación: 09/11/2021

\section{Omar D. Gallo}

Universidad Tecnológica Nacional FR. San Francisco, Argentina

odgallo@gmail.com

\section{Diego M. Ferreyra}

Universidad Tecnológica Nacional FR. San Francisco, Argentina

dferreyra@sanfrancisco.utn.edu.ar

\section{Resumen}

Este estudio trata sobre la graficación de las curvas características, en función de la velocidad, de motores asincrónicos trifásicos de potencias inferiores a $450 \mathrm{~kW}$.

Se usa el circuito equivalente convencional, los datos de ensayos o del fabricante y se procesa la información con lenguaje Python de programación; se calculan impedancias variables, se aplican hipótesis simplificativas, recomendaciones de normas y estimaciones de pérdidas.

Se presentan las devoluciones del programa y algunas de las curvas obtenidas, entre los momentos de arranque y el funcionamiento en vacío. Se agregan las devoluciones bajo distintos estados de carga normalizados y los valores de exigencias eléctricas y magnéticas.

Se pretende aportar un método alternativo para el estudio de motores trifásicos de inducción, con toda la desviación que esto puede incluir, aplicando los principios tradicionales de análisis teórico, los resultados prácticos de los ensayos y un lenguaje moderno de programación.

Palabras claves: circuito equivalente, curvas de motores eléctricos, curvas características de motores eléctricos.

\footnotetext{
Abstract:

This study deals with the plotting of characteristic curves, as a function of speed, of threephase asynchronous motors with power ratings below $450 \mathrm{~kW}$.

Conventional equivalent circuit, test or manufacturer's data are used and the information
} 
is processed with Python programming language; variable impedances are calculated, simplifying assumptions, standard recommendations and loss estimates are applied.

The returns of the program and some of the curves obtained are presented, between startup times and noload operation. The returns under different standardised load states and the values of electrical and magnetic requirements are added.

The aim is to provide an alternative method for the study of threephase induction motors, applying the traditional principles of theoretical analysis, real construction data, practical test results and a modern programming language.

Keywords: equivalent circuit, curves of electric motors, characteristic curves of electric motors.

\section{Introducción}

Uno de los métodos de cálculo de los valores de funcionamiento de un motor eléctrico trifásico consiste en el uso del circuito equivalente (Fraile Mora, 2008: 259-334; Chapman, 2005: 380-431; Liwschitz-Garik y Whipple, 1974: 177-209); este circuito (Fig. 1) está formado por tres ramas: 1) la correspondiente a una fase del primario o bobinado del estator (rama primaria), 2) la correspondiente al comportamiento del circuito magnético (rama derivada) y 3) la correspondiente a una fase del secundario o rotor (rama secundaria), cuyos parámetros están reducidos al primario .

Se aclara que las nomenclaturas de tensiones, impedancias, corrientes, momentos y demás, son propias de este trabajo, y se expresan en las pertinentes referencias.

Referencias de fig. 1 (por fase):

Vf: tensión aplicada, $V$

IIf : corriente de la rama primaria, $A$

$R 1$, jX1: resistencia y reactancia del primario, $\Omega$

E1f: caída de tensión en la rama derivada, $V$

Imf: corriente de la rama derivada (rama del circuito magnético), $A$

$R m$, jXm: resistencia y reactancia de la rama derivada, $\Omega$

I2f: corriente de la rama secundaria; $A$

$R 2$, jX2: resistencia y reactancia del secundario, $\Omega$

E2f: tensión de salida (sobre la carga), $V$

Rcarga: resistencia de carga, $\Omega$

Los parámetros de la rama secundaria son los del rotor, reducidos al estator.

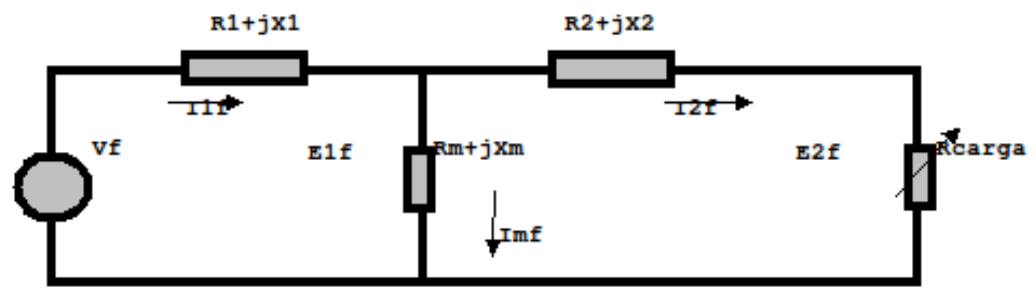

Fig. 1. Circuito equivalente convencional 
Si bien la mayoría de los autores abordan la rama derivada en forma de una resistencia y una reactancia en paralelo, aquí se aplica a ella una única impedancia en serie, porque simplifica el proceso de programación y no afecta los resultados; por lo tanto, la corriente Imf involucra los efectos de magnetización y pérdidas en el hierro.

Cuando se quiere construir este circuito equivalente, se toman resistencia, tensión, corriente, potencia a la entrada y velocidad y momento en el eje durante el arranque, la carga nominal y la marcha en vacío, que se obtienen de ensayos en laboratorio o que son provistas por el catálogo del fabricante (WEG, 2021; LafertGroup, 2021), y se calculan los parámetros $\mathrm{R} 1, \mathrm{X} 1, \mathrm{Rm}, \mathrm{Xm}, \mathrm{R} 2$, X2 en cada rama del circuito. Los valores de impedancias así obtenidos, propios de cada estado de carga, son aproximados porque resultan de aplicar simplificaciones razonables en las ramas.

La bibliografía especializada (Liwschitz-Garik y Whipple, 1974: 227-246) y las experiencias de ensayos (Gallo, 2017), fundamentan que los valores de impedancias indicados son propios para cada estado de carga, porque varían en todas las velocidades (desde el arranque hasta vacío) del motor.

Por lo tanto, para una velocidad cualquiera, el circuito se resuelve disponiendo de R1, X1, $\mathrm{Rm}, \mathrm{Xm}, \mathrm{R} 2$, X2 particulares, determinando la Rcarga = R2 • (1 - s) / s, en donde s es el deslizamiento, y averiguando los valores aproximados en el eje (velocidad, momento y potencia), los de entrada (corriente, potencia y factor de potencia), las corrientes y caídas de tensión en las ramas, las pérdidas y el rendimiento. Frecuentemente, suele ser necesario estimar las pérdidas mecánicas y las adicionales, usando recomendaciones de normas o experiencias prácticas (Norma IEC 60034-1, 2017; Norma IEC 60034-2-1, 2014; EASA/AEMT, 2003: 18-19), para obtener la R2 a partir del momento entregado en el eje.

De aquí que, con miras a incluir las impedancias variables en la resolución del circuito equivalente en todas las velocidades del motor, se asume en este trabajo que:

- la resistencia del primario (R1) es constante con la temperatura

- las reactancias del primario $(\mathrm{X} 1=\omega . \mathrm{L} 1)$ del secundario reducida $(\mathrm{X} 2=\omega . \mathrm{L} 2)$ varían con su coeficiente de autoinducción $\left(\mathrm{L}=\mathrm{N} . \Phi_{\delta} / \mathrm{I}\right)$, que depende del flujo disperso $\left(\Phi_{\delta}\right)$ y la corriente (I) variables. $\mathrm{N}$ son las espiras involucradas (Corrales Martín I, 1976: 210-214).

- la resistencia $(\mathrm{Rm})$ de la rama derivada (efecto de histéresis y corrientes parásitas), se considera constante, suponiendo que se mantienen aproximadamente estables las condiciones de magnetización del hierro (Chapman, 2005: 21-28).

- la reactancia de la rama derivada (Xm) varía con la corriente de magnetización (Corrales Martín I: 409-411).

- el coeficiente de autoinducción de los conductores del rotor y por lo tanto su resistencia equivalente (R2) varía con el flujo disperso concatenado (Corrales Martín I, 1976: 246-250).

El objetivo aqui planteado es, admitiendo tal variación de las impedancias con la velocidad en el circuito equivalente, obtener las curvas de las principales magnitudes de entrada y salida del motor eléctrico, desde el instante de arranque, hasta su marcha en vacío.

Para satisfacer este objetivo, se elabora un software específico basado en datos de ensayos y fabricación, que anexa además la obtención de exigencias eléctricas y magnéticas en 
condiciones nominales y datos de curvas proporcionados por el fabricante.

Existen numerosas publicaciones referidas o relacionadas con el tema propuesto. Los aspectos generales de algunas de ellas se abordan brevemente a continuación.

Sale A. et al. (2018) utiliza un método similar al aquí propuesto, llamado método de ingeniería para optimización de parámetros de momento en el eje y corriente, en varios motores normales y usando datos del fabricante. El análisis y la resolución del circuito equivalente son distintos, no se entregan resultados de corrientes o caídas de tensión en las ramas y se realiza la misma comparación de curvas de momentos y corrientes, con buenos resultados.

Wengerkievicz C. et al. (2017) presenta un estudio en el cual se considera el circuito equivalente y la variación de los parámetros por efecto de la saturación. Estudia los métodos alternativos propuestos por varios autores y compara sus resultados con los del fabricante. Presenta comparaciones de impedancias y potencias variables. No muestra valores de magnitudes en las ramas del circuito, ni entrega comparaciones de curvas de momentos y corriente.

Amaral, G.F.V et al. (2021) presenta un estudio de circuito equivalente de motores desde $1 \mathrm{~kW}$ a $520 \mathrm{~kW}$ de tres marcas usando los valores del folleto del fabricante y arribando, con buenos resultados, a los valores requeridos de $50 \%$, $75 \%$ y $100 \%$ de carga. Tampoco entrega valores internos en las ramas del circuito, para cada velocidad.

En linea también se encuentran disponibles múltiples aplicaciones comerciales o de libre acceso que apuntan al diseño o reconstrucción de motores eléctricos, que se comentan seguidamente.

Indielec, siglas de la empresa Ingeniería de diseño electrotécnico. ofrece software que aplica diseño asistido por computadora (CAD) para esquemas de devanados, cálculo de refrigeración y dimensionamiento preliminar de motores eléctricos (https://www.indielec. com/software-para-maquinas-electricindielec-cms-4-50-494/)

Multisim es una aplicación destinada generalmente a usos electrónicos, que se puede aplicar para armar el circuito equivalente del motor de inducción, colocando instrumental en cada rama y obteniendo los valores requeridos en cada estado de velocidad. (https:// www.multisim.com/

Progebo, siglas de Programa de gestión de bobinados, es una plataforma interactiva de acceso libre que permite calcular, dibujar, compartir y almacenar datos de arrollamientos trifásicos de motores de inducción (http://www.progebo.com/)

En resumen, se evidencia que las investigaciones nombradas comparten algunos principios y criterios con este trabajo y arriban a similares resultados, inclusive mediante el uso de otros métodos. Sin embargo, no muestran valores de las ramas internas del circuito equivalente, que son necesarios para obtener valores de flujo principal, corriente de magnetización y discriminación de pérdidas.

Por otro lado, las aplicaciones comerciales o de libre acceso, se refieren preferentemente al diseño físico, esquemas o dibujos de la máquina y la comprobación de su comportamiento durante la marcha.

Por su parte, este trabajo pretende aportar un método alternativo para el análisis del comportamiento de motores de inducción, compartiendo diversos aspectos con lo disponible en red y anexando la obtención de otros valores que en las publicaciones no se presentan.

\section{Desarrollo}

En la elaboración del software se utiliza Python 3 (Python S.F., 2021) porque presenta, entre otras, las ventajas de ser un lenguaje de programación de última generación, accesible 
en la red, actualizable, de sintaxis sencilla, multiplataforma, y con las posibilidades de extenderlo, eventualmente, al dominio de la inteligencia artificial.

En esta etapa inicial del desarrollo, esta aplicación reúne los siguientes requisitos elementales:

- Sencillez de comunicación con el usuario, rápida carga y ágil lectura de datos: posee una interfaz amigable y velocidad de comunicación, lo que posibilita un pronto y sencillo acceso a los resultados.

- Posibilidad de graficar, almacenar, recuperar y exportar los valores: los resultados se muestran en tablas y gráficos e incluye soporte y detalle de los datos almacenados. Los valores obtenidos pueden exportarse a una planilla de cálculo estándar.

- Aplicación a motores trifásicos de inducción de hasta $450 \mathrm{~kW}$, en baja tensión: los principios físicos y matemáticos considerados se acotan a este tipo de máquinas y rango de potencias, porque son los datos que se disponen.

- Libre acceso en la red: factibilidad de usarse, adaptarse o actualizarse en el ámbito profesional o académico, sin costos económicos.

- Algunas de las ventajas del uso de este software son:

- Desde la visión del usuario, posibilita aplicar el método de resolución del circuito equivalente convencional para obtener todas sus magnitudes de funcionamiento en toda la extensión de la curva momento \& velocidad, disponiendo solamente de datos de identificación, arranque, carga nominal y vacío del motor. Esto constituye una prestación muy valorada, porque facilita la realización de sucesivos ajustes en el diseño y fabricación de líneas de motores eléctricos, extiende los resultados de los ensayos a otros estados de carga o velocidades no registrados en el laboratorio e incluso posibilita adaptar las curvas de un prototipo a exigencias de arranque o variación de velocidad especiales.

- Para los autores en particular, es una herramienta que se usará para realizar futuras investigaciones en series completas de motores ya fabricados y ensayados (como más adelante se muestra en las tablas y figuras comparativas), con la finalidad de originar bibliografía de referencia y agregarla a la biblioteca de la plataforma Progebo, ya mencionada (Gallo y Gallo, 2014, 2017).

Los siguientes requisitos de uso están integrados en este software: a) ingreso de datos iniciales del motor, b) ingreso de datos de resistencias de arrollamientos y ensayos en vacío, arranque y estados de carga (100\%, 75\% y 50\%), c) obtención de valores iniciales (preliminares), d) obtención de valores en función de la velocidad, e) obtención de valores característicos de la curva, f) comparación con datos de ensayos o del fabricante, g) ingreso de datos constructivos y cálculo de exigencias y h) exportación de valores a planillas de cálculo $\mathrm{u}$ otros archivos. Seguidamente se detallan cada uno de ellos.

a) Ingreso de datos iniciales del motor: se ingresan y confirman los datos identificatorios y característicos del motor, requeridos en posteriores cálculos. Se muestra, como ejemplo, la devolución del software, para un motor de $75 \mathrm{~kW} \mathrm{y} 4$ polos, en tabla 1. 
Referencias de tabla 1:

Cód/N $N^{\circ}$ código o número del motor

Tipo: tamaño constructivo (altura del eje ). S: short, $M$ : medium, L: large

$k W$ : potencia entregada en el eje, $k W$

$V$ : tensión de alimentación, $V$

Conn: conexión del motor. T: triángulo

$\mathrm{Hz}$ : frecuencia, $\mathrm{Hz}$

min-1: velocidad nominal, min-1

Eff: eficiencia, \%

Fdp: factor de potencia

A: corriente nominal, $A$

Ia/In: relación corriente de arranque/corriente nominal

$\mathrm{Ma} / \mathrm{Mn}$ : relación de momentos de arranque/momento nominal

$\mathrm{Mm} / \mathrm{Mn}$ : relación de momento máximo/momento nominal

Aisl: clase térmica de los aislantes (F: $\left.155^{\circ} \mathrm{C}\right)$

IP: tipo de protección

Especialidad: alguna característica especial del motor

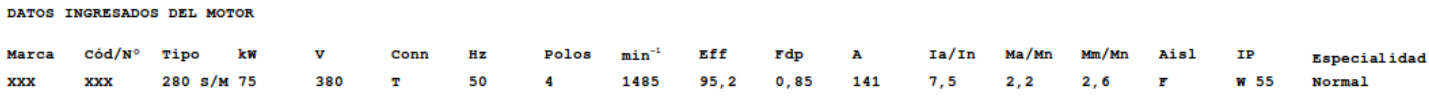

Tabla 1. Devolución de datos identificatorios de un motor de 75 kW, 4 polos

b) Ingreso de datos de resistencias de arrollamientos y ensayos en vacío, arranque y estados de carga (100\%, 75\% y 50\%): se ingresan y confirman los datos de resistencias óhmicas en frío y en caliente y las tensiones, corrientes, potencias, momentos, velocidades y temperaturas, obtenidos de ensayos (devolución en tabla 2). Según las normalizaciones, se fija la temperatura de cálculo $\left(25^{\circ} \mathrm{C}\right)$ y se estiman las pérdidas mecánicas y adicionales (Norma IEC 60034-2-1, 2014).

Referencias de tabla 2:

Resistencia frío / cal: resistencia de una fase del bobinado, en frío / estabilizado térmicamente Tambte frio / cal: temperatura del ambiente, en frío / estabilizado

Sobretemperatura: promedio de temperatura del bobinado sobre la del ambiente (método por variación de resistencia)

Vacio, arranque y carga al 75\% y 50\% (de la nominal): valores reales de ensayos

Temp carcasa: temperatura en un punto determinado de la carcasa, estabilizado en temperatura

Tens: tensión aplicada

$n x$ : velocidad

Mej: momento en el eje, $\mathrm{Nm}$

P1t: potencia de entrada, $W$

Pej: potencia en el eje, $W$

Ili: corriente de linea, $A$ 


\begin{tabular}{|c|c|c|c|c|c|c|c|c|c|c|}
\hline Ensayo & $(\Omega)$ & $\left({ }^{\circ} \mathrm{C} / \mathrm{K}\right)$ & Tens (V) as & $\left(\min ^{-1}\right)$ & $\operatorname{Mej}(\mathrm{Nm})$ & $\operatorname{Eff}(8)$ & P1t (w) & $\operatorname{Pej}$ (w) & $\operatorname{Ili}(\mathrm{A})$ & Fdp \\
\hline Resistencia frio & 0,0400 & -- & -- & -- & -- & -- & -- & -- & -- & -- \\
\hline Tambte frio & -- & 24,9 & -- & -- & -- & -- & -- & -- & -- & -- \\
\hline Resistencia cal. & 0,0487 & & -- & -- & -- & -- & -- & -- & -- & - \\
\hline Tambte cal & -- & 24,9 & -- & -- & -- & -- & -- & -- & -- & -- \\
\hline sobretemperatura & -- & 56,5 & -- & -- & -- & -- & -- & -- & -- & -- \\
\hline Vacio & -- & -- & 380 & 1500 & -- & -- & 1357 & -- & 50,6 & 0,04 \\
\hline Arranque & -- & -- & 380 & 0 & 1061,0 & -- & -- & -- & 1057,5 & -- \\
\hline Carga $75 \%$ & -- & -- & 380 & 1490 & 360,7 & 95,4 & 58962 & 56252 & 113,0 & 0,79 \\
\hline Carga 508 & -- & -- & 380 & 1493 & 240,0 & 95,0 & 39492 & 37504 & 85,0 & 0,71 \\
\hline emp. Carcasa & -- & 79,0 & -- & -- & -- & -- & -- & -- & -- & - \\
\hline
\end{tabular}

Tabla 2. Devolución de datos de arrollamiento y ensayos, 75 kW, 4 polos

c) Obtención de valores iniciales (preliminares): se obtienen, en primer lugar, los valores preliminares de impedancias durante el arranque y en carga nominal eliminando la rama derivada $(\mathrm{Rm}, \mathrm{Xm})$ e igualando las reactancias del primario y secundario. Luego se calculan las impedancias en vacío y se recalculan las impedancias nominales, incluyendo esta vez la rama derivada (Tablas 3 y 4). Los de arranque y nominales son los parámetros iniciales que se usarán en las funciones de variación de X1, X2 y R2, expresadas en el programa del punto d).

Referencias de tablas 3 y 4:

Arranque: valores de arranque obtenidos sin considerar la rama derivada del circuito $(\operatorname{Imf}=0)$

Nominal1 : valores nominales obtenidos sin considerar la rama derivada del circuito

Vacío: valores obtenidos considerando la rama derivada, con reactancias y resistencia rotórica variables

Nominal2: recálculo de valores nominales, con parámetros variables y rama derivada, según proceso del punto d)

$R t$, Xt: resistencia y reactancia total del circuito, $\Omega$

$R 1, X 1$ : resistencia y reactancia del primario, $\Omega$

$R m, X m$ : resistencia y reactancia de la rama derivada, $\Omega$

Imf, Iml: corrientes por fase y de línea, A; Im>: ángulo de la corriente respecto a la tensión, ${ }^{\circ}$

$R 2, X 2$ : resistencia y reactancia del secundario, $\Omega$

P1t: potencia de entrada, $W$

Pad, Pme: pérdidas adicionales y mecánicas, respectivamente, $W$

Pfe, Pcu: pérdidas en el hierro y térmicas, $W$

E2f: tensión de salida (a bornes de la carga), V; E2>: ángulo entre E2fy la tensión de entrada, ${ }^{\circ}$

E1f: caída de tensión sobre la rama derivada, $V$ : E1>: ángulo de E1, ${ }^{\circ}$

I2f: corriente de la rama secundaria, A; I2>: ángulo de I2f, ${ }^{\circ}$

Ilf: corriente de la rama primaria, $A$

I1l: corriente de linea del motor, A; I1>: ángulo de I1f, ${ }^{\circ}$ 


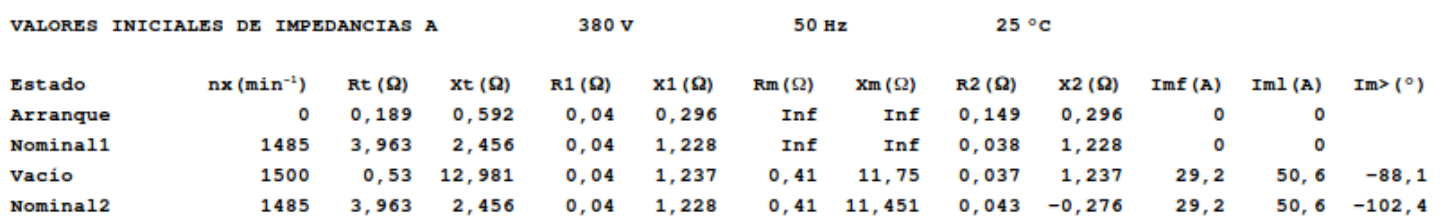

Tabla 3. Devolución de valores iniciales de impedancias, 75 kW, 4 polos

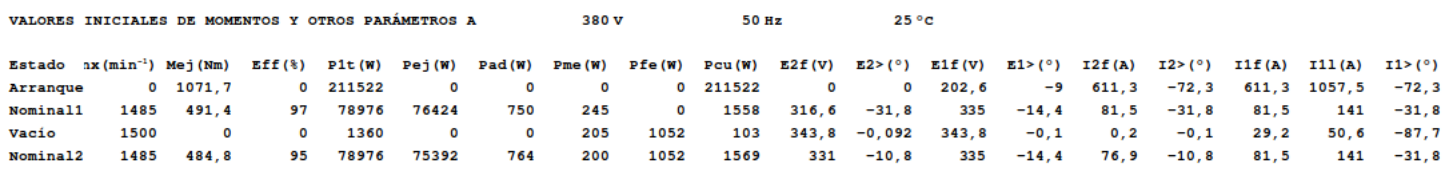

Tabla 4. Devolución de valores de iniciales de momentos y otros, 75 kW, 4 polos

d) Obtención de valores en función de la velocidad: se utiliza un método de cálculo similar al del punto c) con rama derivada incluida, pero en este caso, las reactancias y la resistencia rotórica se consideran variables con la velocidad (Corrales Martín I, 1976: 236-276; Juha Pyrhönen et al. , 2008: 225-280, Chapman, 2005: 416-423), y se admite que son invariables la resistencia del primario y la de la rama derivada.

Seguidamente, se muestran algunas de las líneas de instrucciones de esta parte del programa, con sus aclaraciones. La devolución de los valores calculados se muestra en las tablas 5 y 6 .

Referencias sobre el programa de obtención de valores en función de la velocidad:

Cad: Coeficiente de pérdidas adicionales, estimadas según recomendaciones de norma

E10: Caída de tensión en la rama derivada, en vacio (proceso del punto c), $V$

E1a: Caída de tensión en la rama derivada, durante el arranque (proceso del punto c), $V$

IlIf: relación entre corriente de línea y corriente de fase

kmx: coeficiente de variación de la reactancia derivada. Es un valor estimado

n: velocidad nominal (dato de ensayo o del fabricante), 1/min

ns: velocidad sincrónica, 1/min

Pmeco: Pérdidas mecánicas en vacio, estimadas en un porcentaje de la potencia en vacio, $W$

R0: resistencia total del circuito, en vacio (proceso del punto c), $\Omega$

$R 1 f$ : resistencia por fase a la temperatura establecida (dato de ensayo), $\Omega$

$R 2 a$ : resistencia reducida del rotor, según datos de arranque (proceso del punto $c$ ), $\Omega$

$R c 0$ : resistencia de la carga en vacio, por pérdidas mecánicas (proceso del punto c), $\Omega$

$R t 2 n$ : resistencia reducida del rotor, según datos nominales (proceso del punto c), $\Omega$

V1n: tensión nominal, $V$

$X 0$ : reactancia total del circuito, en vacio (proceso del punto c), $\Omega$

$X 1$ a: reactancia del primario, según los datos de arranque (proceso del punto c), $\Omega$

$X 2 a$ : reactancia reducida del primario, según datos de arranque (proceso del punto c), $\Omega$

$X t 1 n$ : reactancia del primario, según datos nominales (proceso del punto c), $\Omega$

$X t 2 n$ : reactancia reducida del rotor, según datos nominales (proceso del punto c), $\Omega$

Zm0: impedancia de la rama derivada, en vacio (proceso del punto c), $\Omega$

Preliminarmente, la ley de variación de resistencias y reactancias ( $\mathrm{R} 2 \mathrm{x}, \mathrm{X} 1 \mathrm{x}, \mathrm{X} 2 \mathrm{x})$ con la velocidad se considera una función lineal entre arranque y nominal. Posteriormente, esta 
ley podrá ser ajustada a la curva del fabricante.

Los valores redondeados son los que se muestran en las devoluciones.

\section{\# PROGRAMA DE OBTENCIÓN DE VALORES EN FUNCIÓN DE LA VELOCIDAD}

for $m$ in range $(1,3)$ :

for $n x$ in range $(0,(n s+1), 1)$ :

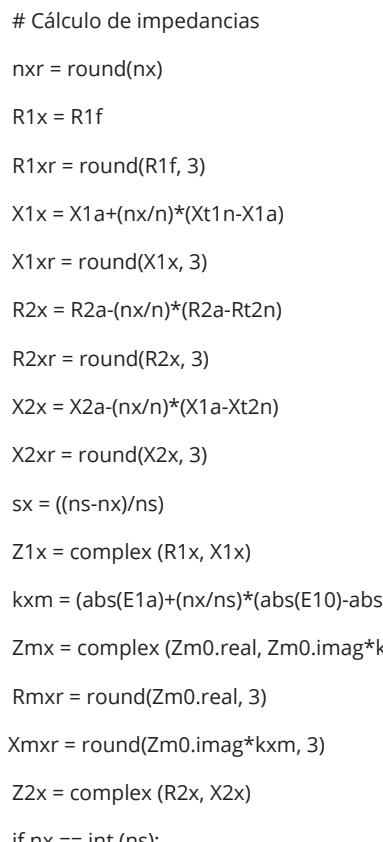

if $n x==$ int (ns):

$\mathrm{Z} 22 \mathrm{x}=$ complex $((\mathrm{R} 2 \mathrm{x}+\mathrm{Rc} 0), \mathrm{X} 2 \mathrm{x})$

$\mathrm{Ztx}=\operatorname{complex}(\mathrm{RO}, \mathrm{X} 0)$

else:
\# Repetición del proceso

\# Rango de velocidad (nx) de cálculo, 0 a ns

\# $\mathrm{nx}$ redondeada (1/min)

\# Resistencia del primario (constante)(W)

\# R1x redondeada, 3 decimales

\# Reactancia variable del primario

\# $\mathrm{X} 1 \mathrm{x}$ redondeada

\# Resistencia variable del secundario (rotor)

\# R2x redondeada

\# Reactancia variable del secundario

\# $\mathrm{X} 2 \mathrm{x}$ redondeada

\# Deslizamiento variable

\# Complejo impedancia del primario

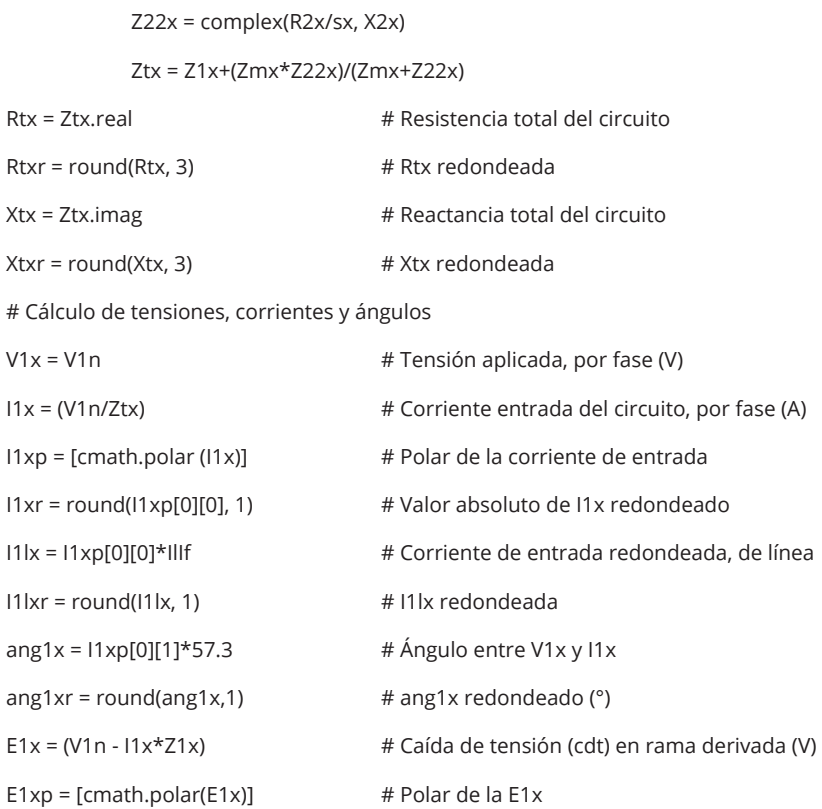

$$
\begin{aligned}
& \mathrm{Z} 22 \mathrm{x}=\operatorname{complex}(\mathrm{R} 2 \mathrm{x} / \mathrm{sx}, \mathrm{X} 2 \mathrm{x}) \\
& \mathrm{Ztx}=\mathrm{Z} 1 \mathrm{x}+\left(\mathrm{Zm} \mathrm{x}^{\star} \mathrm{Z} 22 \mathrm{x}\right) /(\mathrm{Zmx}+\mathrm{Z} 22 \mathrm{x})
\end{aligned}
$$




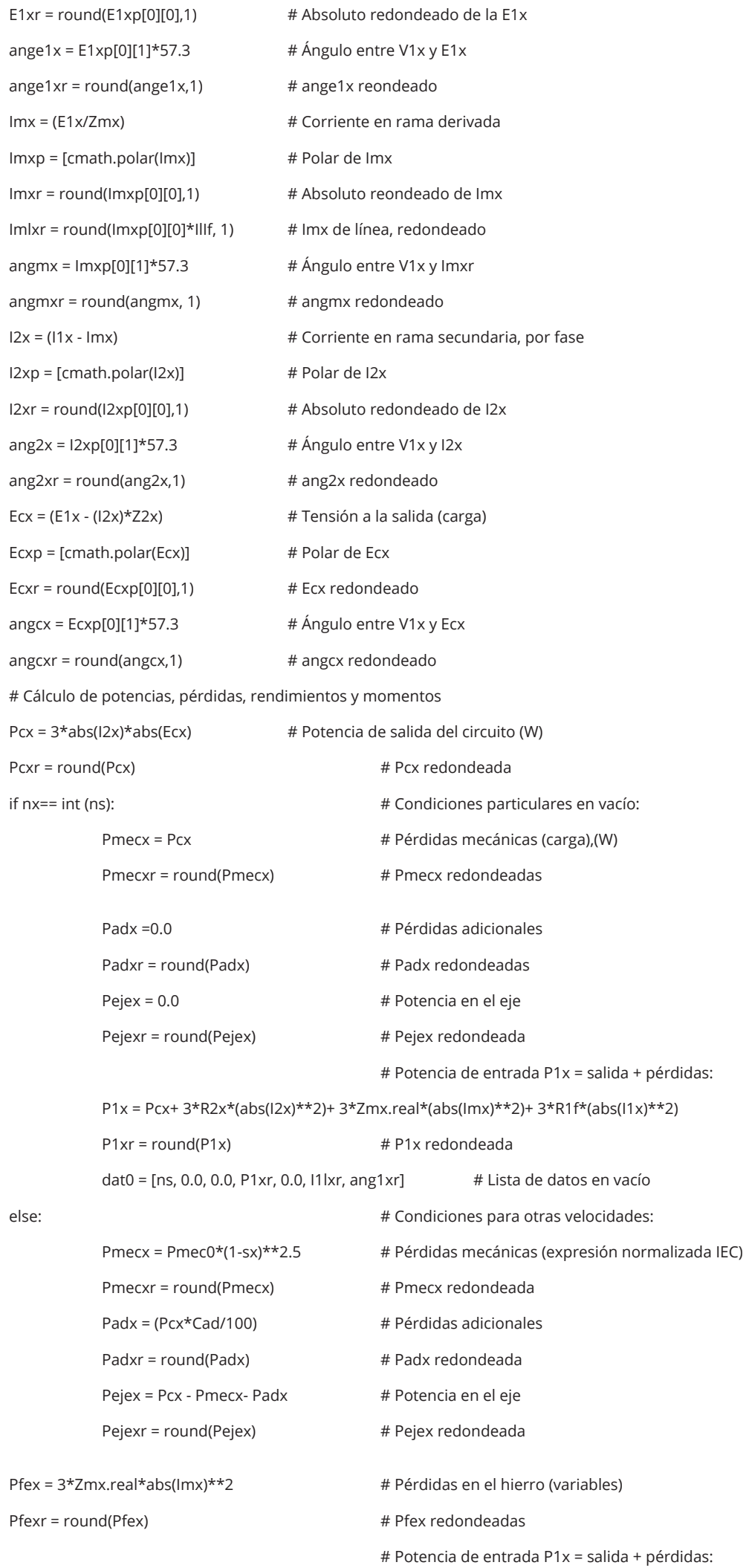




\begin{tabular}{|c|c|}
\hline $\mathrm{P} 1 \mathrm{xr}=\operatorname{round}(\mathrm{P} 1 \mathrm{x})$ & \# P1x red \\
\hline Pcux $=3 *\left(R 1 f^{\star} a b s(11 x)^{\star \star} 2+R 2 x^{*} a b s(12 x)^{\star \star} 2\right)$ & \# Pérdid \\
\hline \multicolumn{2}{|l|}{ Pcuxr $=$ round $($ Pcux $)$} \\
\hline \multicolumn{2}{|l|}{ Eff $x=100 *$ Pejex $/ P 1 x$} \\
\hline \multicolumn{2}{|l|}{ Effxr $=\operatorname{round}(E f f x, 1)$} \\
\hline \multicolumn{2}{|l|}{ if $n x==0$ : } \\
\hline \multicolumn{2}{|c|}{$M x=60^{*} 3^{*}(\mathrm{R} 2 \mathrm{x} / \mathrm{sx})^{\star} \mathrm{abs}(12 \mathrm{x})^{\star *} 2 /\left(2^{*} \pi^{*} \mathrm{~ns}\right)$} \\
\hline \multicolumn{2}{|l|}{$M x r=\operatorname{round}(M x, 1)$} \\
\hline \multicolumn{2}{|c|}{$\operatorname{dat} A=[0.0, M x r, ' 0.0$ ', P1xr, Pejexr, $111 \times r$, ang1xr] } \\
\hline \multicolumn{2}{|l|}{ else: } \\
\hline \multicolumn{2}{|l|}{$M x=60^{*}$ Pejex $/\left(2^{*} \pi^{*} n x\right)$} \\
\hline$M x r=\operatorname{round}(M x, 1)$ & \\
\hline
\end{tabular}

IMPEDANCIAS EN FUNCIÓN DE LA VELOCIDAD A $\mathrm{ax}\left(\mathrm{min}^{-1}\right) \quad \mathrm{Rt}(\Omega) \quad \mathrm{xt}(\Omega) \quad \mathrm{R} 1(\Omega) \quad \mathrm{x} 1(\Omega)$

$\begin{array}{rllll}0 & 0,177 & 0,582 & 0,040 & 0,296\end{array}$

$60 \quad 0,180 \quad 0,599 \quad 0,040 \quad 0,334$

$120 \quad 0,183 \quad 0,615 \quad 0,040 \quad 0,371$

$\begin{array}{rrrrr}\ldots . . . & \ldots & \ldots & \ldots \\ 1380 & 0,700 & 0,960 & 0,040 & 1,162\end{array}$

$1440 \quad 1,244 \quad 1,060 \quad 0,040 \quad 1,200$

$1500 \quad 0,530 \quad 12,981 \quad 0,040 \quad 1,237$
\# Eficiencia

\# Effx redondeada

\# Condiciones en el arranque:

$$
\begin{aligned}
& \text { \# Momento en el eje ( } \mathrm{Nm}) \\
& \text { \# Mx redondeado } \\
& \text { \# Datos de arranque }
\end{aligned}
$$

\# Condiciones para otras velocidades:

\# Momento en el eje

\# Mx redondeado

Tabla 5. Resumen de impedancias en función desde el arranque hasta vacío, 75 kW, 4 polos

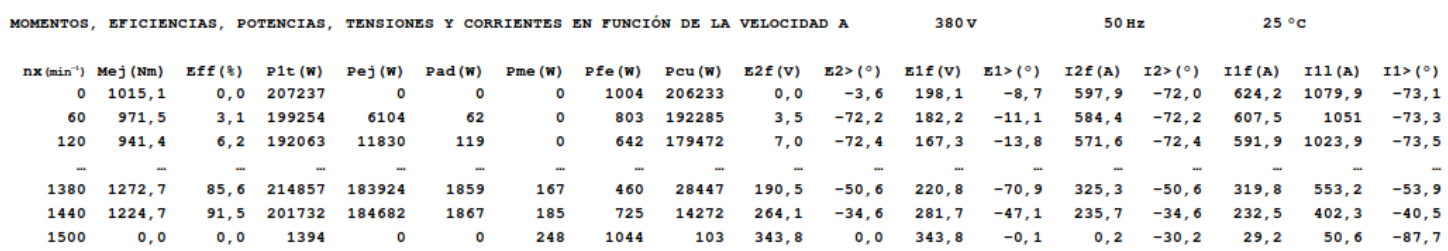

Tabla 6. Resumen de devolución de momentos y otros desde el arranque hasta vacío, 75 kW, 4 polos

Si bien el programa resuelve para todas las velocidades enteras, desde 0 a ns, sólo se devuelven normalmente 26 líneas de valores, desde 0 a ns y a intervalos enteros ns/25, de los que la tabla 6 muestra tres iniciales y tres finales. No obstante, se puede elegir otro intervalo distinto de ns/25.

e) Obtención de valores característicos de la curva: el programa obtiene los valores de momento mínimo y máximo y la respuesta a 150\%, 125\%, 100\%, 75\%, 50\% y 25\% de carga, además de la respuesta a máximo rendimiento (devolución en tabla 7).

Los valores a distintos estados de carga se obtienen por interpolación, de manera tal que la potencia entregada en el eje sea exactamente igual al valor indicado por los porcentajes de carga.

f) Comparación con datos de ensayos o del fabricante: se comparan los datos obtenidos por cálculo con los ingresados de los ensayos de estados de carga y/o con los valores 
de las curvas del fabricante. La devolución de estos valores se mostrará en la sección resultados y discusión

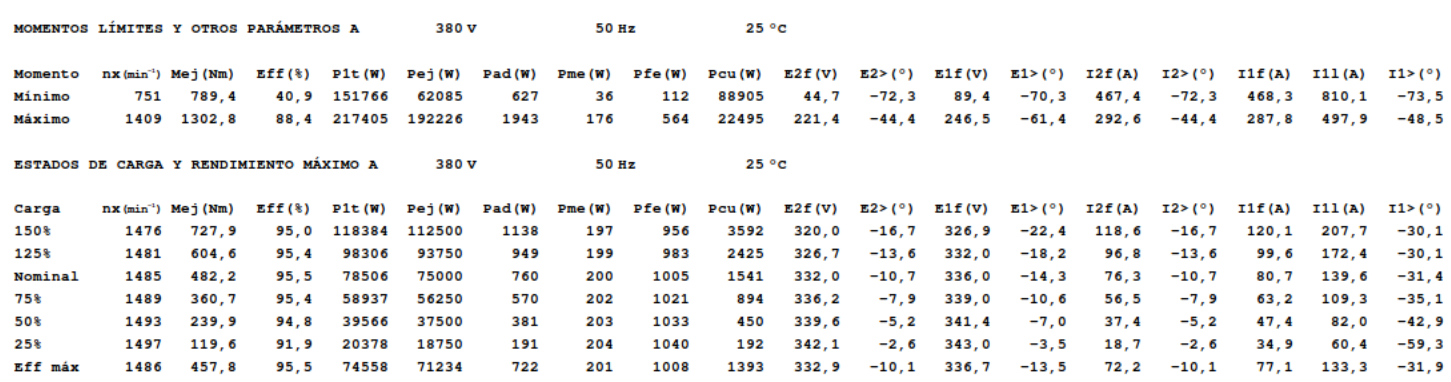

Tabla 7. Devolución de valores característicos, 75 kW, 4 polos

g) Ingreso de datos constructivos y cálculo de exigencias: si se encuentran disponibles, los datos dimensionales del paquete de chapas y los del bobinado permiten acceder al cálculo aproximado de las exigencias eléctricas, magnéticas y térmicas en condiciones nominales. Ésto constituye un agregado a la prestación principal del software, que es la obtención de curvas, y se implementó porque resulta ser una herramienta de gran utilidad para los diseñadores de motores.

Seguidamente, se muestran algunas de las líneas de instrucciones de esta parte del programa, con sus aclaraciones. Los valores finales son aproximados y redondeados.

Referencias sobre el programa de cálculo de exigencias:

Cap: capas de bobinas por ranura

Cbo: ramas en paralelo del bobinado

Dej: diámetro del eje, $\mathrm{mm}$

Dro: diámetro interior del estator (aproximadamente del rotor), $\mathrm{mm}$

E1: cdt en la rama derivada, $V$

Ebo: espiras por bobina (promedio)

Eh: longitud del entrehierro, $\mathrm{mm}$

$f:$ frecuencia, $\mathrm{Hz}$

Fap: factor de apilado de las chapas

Far: factor de arrollamiento

Hce: altura de la corona del estator, $\mathrm{mm}$

Hcr: altura de la corona del rotor, $\mathrm{mm}$

Heh : intensidad de campo magnético en el entrehierro, en vacio, $A / m$

Im0: corriente derivada, en vacio, $A$

In: corriente nominal, $A$

Lpa: longitud del paquete de chapas, $\mathrm{mm}$

Nre: Cantidad de ranuras del estator

Nrr: Cantidad de ranuras del rotor

P1e: potencia de entrada, estabilizado térmicamente, $W$

Pejee: potencia en el eje, estabilizado térmicamente, $W$

$P n$ : potencia nominal, $k W$

polos: cantidad de polos 
Sar: sección de los anillos del rotor, $\mathrm{mm}^{2}$

Scu: sección de conductor, por rama, $\mathrm{mm}^{2}$

tc: temperatura del ambiente cuando ocurre la estabilización térmica, ${ }^{\circ} \mathrm{C}$

tcare: temperatura de la carcasa del motor, estabilizado térmicamente, ${ }^{\circ} \mathrm{C}$

TW: sobretemperatura del arrollamiento en relación a las pérdidas, ${ }^{\circ} \mathrm{C} / \mathrm{W}$

\# PROGRAMA ANEXO DE CÁLCULO DE EXIGENCIAS

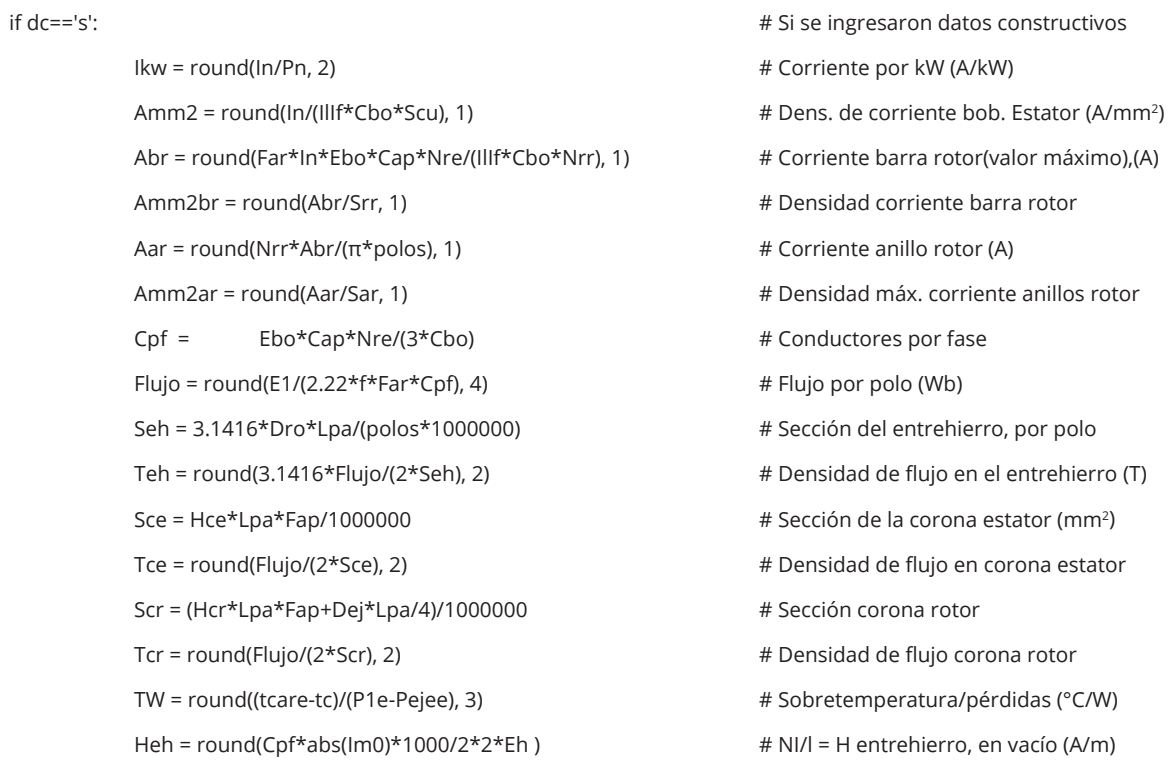

La devolución de datos constructivos y el cálculo de exigencias de un motor similar al indicado en el punto a), pero de otro fabricante, se muestran en la tabla 8.

\begin{tabular}{|c|c|c|c|c|c|}
\hline Dimensiones & Datos dim. & Bobinado & Datos bob. & Exigencias & Valores exig. \\
\hline Diámetro exterior estator (mm) & 430,5 & Paso de bobinas en ranuras & $10 / 12$ & Corriente por $\mathrm{kW}(\mathrm{A} / \mathrm{kW})$ & 1,88 \\
\hline Altura corona estator $(\mathrm{mm})$ & 47,8 & Capas de bobinas por ranura & 1 & Densidad corriente bobinado $(\mathrm{A} / \mathrm{mm} 2)$ & 4,5 \\
\hline Altura corona rotor (mm) & 43,7 & Espiras por bobina (prom) & 11 & Densidad corr, barra rotor $(\mathrm{A} / \mathrm{mm} 2)$ & 3,0 \\
\hline Diámetro eje rotor (mm) & 95 & Espiras por bobina (real) & $11 / 11$ & Corriente anillo rotor & 1966,2 \\
\hline Longitud paquete de chapas (mm) & 330 & Número de alambres 1 & 7 & Densidad corr. anillo rotor $(\mathrm{A} / \mathrm{mm} 2)$ & 3,0 \\
\hline Número ranuras estator & 48 & Diämetro alambre 1 (mm) & 1,4 & Flujo por polo (wb) & 0,0365 \\
\hline sección ranura rotor ( $\mathrm{mm} 2$ ) & 207,7 & conexion bobinado (vias) & 2 & Dens. flujo corona rotor (T) & 0,88 \\
\hline Espesor anillo rotor (mm) & 15 & Peso total cobre $(\mathrm{kg})$ & 35,1 & sobretemperat. carcasa/pérdidas $\left({ }^{\circ} \mathrm{K} / \mathrm{W}\right)$ & 0,005 \\
\hline sección anillo rotor $(\mathrm{mm} 2)$ & 657 & Relleno ranura (₹) & 38,9 & $\mathrm{NI} / 1=\mathrm{H}$ entrehierro, vacio $(\mathrm{A} / \mathrm{m})$ & 2601160 \\
\hline Longitud entrehierro (mm) & 0,8 & & & & \\
\hline
\end{tabular}

Tabla 8. Devolución de datos constructivos y cálculo de exigencias, 75 kW, 4 polos (otro fabricante)

h) Exportación de valores: además de la devolución en pantalla de los datos ingresados y obtenidos, éstos se exportan a una planilla de cálculo estándar, en donde se obtienen las curvas características.

\section{Resultados y discusión}

Los resultados del método expuesto se exponen seguidamente, abordando los siguientes aspectos : a) comparación con datos de ensayos: comparación de los valores obtenidos por 
el software en el arranque, en los puntos de momento mínimo y/o máximo, y los estados de carga, respecto a los datos obtenidos de ensayos, b) comparación con datos de la curva del fabricante: comparación de los valores en distintos tramos de la curva de momentos y corrientes obtenida, con respecto a los mismos datos, dados por el fabricante c) graficación de curvas de las distintas magnitudes: construcción y evaluación de las curvas de las distintas magnitudes calculadas y d) elaboración de tablas comparativas anexas: opcionalmente, se confeccionan tablas útiles para los proyectistas de máquinas.

a) Comparación con datos de ensayos: La comparación con datos de ensayos o del fabricante (punto f del título anterior), para el motor de $75 \mathrm{~kW}$, devuelve los resultados mostrados en tabla 9.

Si bien también se muestran aquí los valores absolutos, las diferencias entre los valores reales (ingresados) y los calculados siempre se tomarán en porcentaje, a los fines de facilitar la rápida evaluación de los resultados logrados.

Las mayores diferencias (tabla 9) se producen en el momento (4,33 \%) y la corriente $(-2,12 \%)$ durante el arranque; en condiciones nominales, la mayor diferencia es en el ángulo (1,23\%), y en el $75 \%$ y 50 \% de carga, también las mayores diferencias están en los ángulos. En vacío, la diferencia en corrientes es del -2,73 \%.

El proceso se repitió con otros motores de 4 polos, igual tensión y frecuencia, todos del mismo fabricante (tabla 10); se tomó un motor de potencia mucho menor (tamaño $112 \mathrm{M}, 4 \mathrm{~kW}$ ) y otro de potencia algo mayor (tamaño $315 \mathrm{~S} / \mathrm{M}, 132 \mathrm{~kW}$ ). Cabe observar que en estos cálculos se conservaron las estimaciones de pérdidas mecánicas ( $20 \%$ de las del hierro), pérdidas adicionales (1\% de la potencia de salida) y las leyes de variación de las resistencias y reactancias con la velocidad.

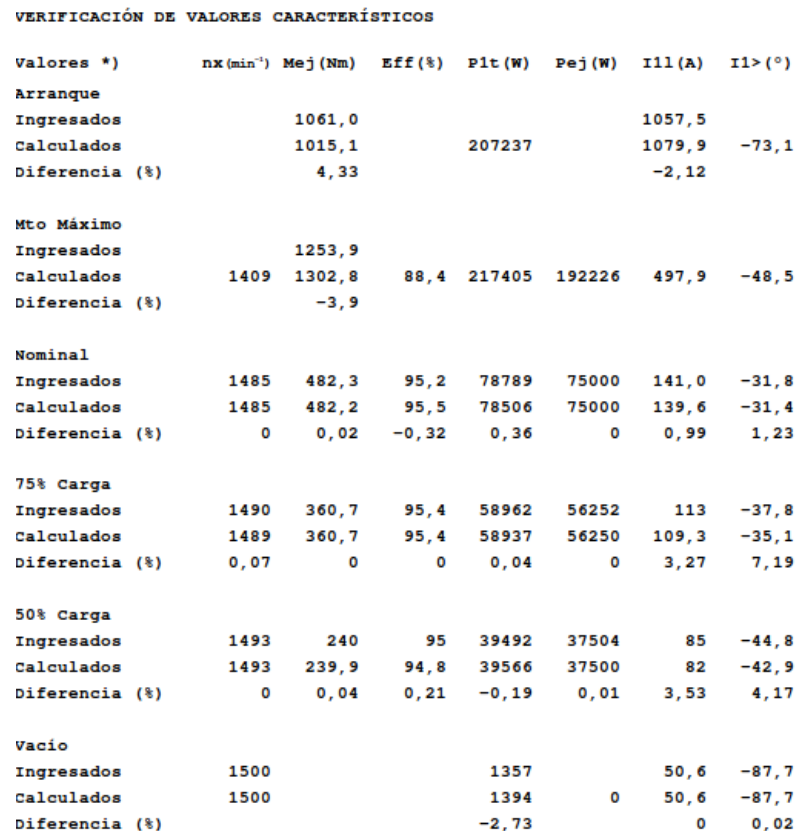

Tabla 9. Comparativa de estados de carga, $75 \mathrm{~kW}$ 


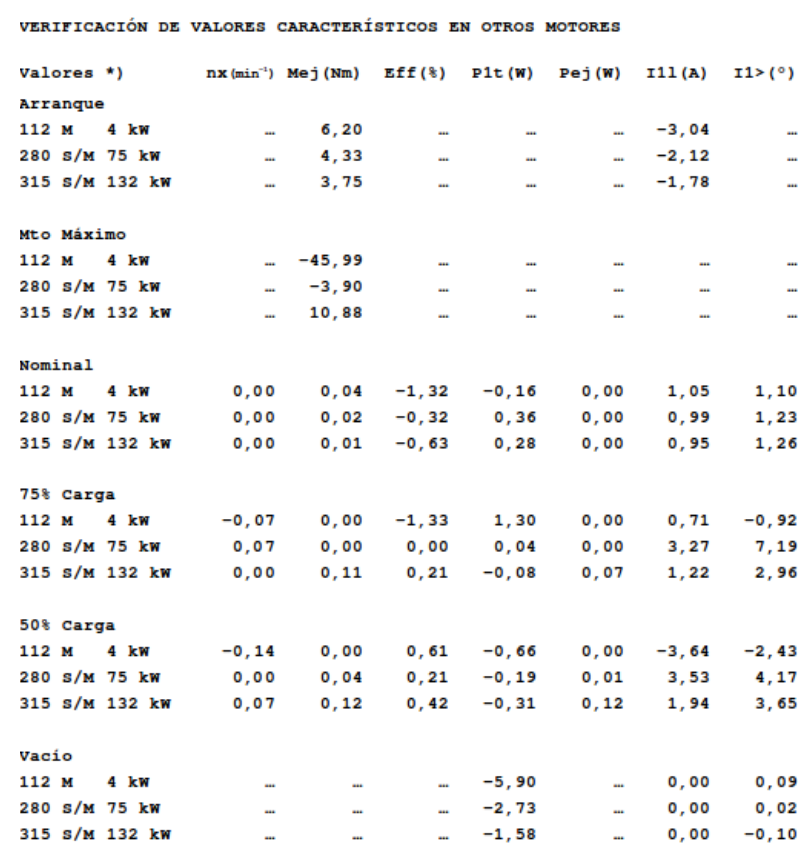

Tabla 10. Diferencias en tres motores

En dichas tablas 9 y 10, se observa que algunas diferencias de valores, respecto al motor de $75 \mathrm{~kW}$, se hacen ligeramente superiores en el de $132 \mathrm{~kW}$ y se acentúan las diferencias en el de $4 \mathrm{~kW}$ (que está bastante alejado de los otros en potencia). Se infiere que para reducir las diferencias importantes entre algunos ítems, será necesario ajustar las estimaciones de pérdidas mecánicas y adicionales a cada potencia en particular.

b) Comparación con datos de la curva del fabricante: La comparación de los valores en distintos tramos de la curva de momentos, para $75 \mathrm{~kW}$, arroja los resultados de la Fig. 2.

Referencias de la Fig. 2:

Columna A: velocidad, min-1

Columna B: Momento según curva del fabricante (fab), Nm

Columna C: Momento curva de cálculo (calc), Nm

Columna D: Diferencia $100 *(B-C) / B$
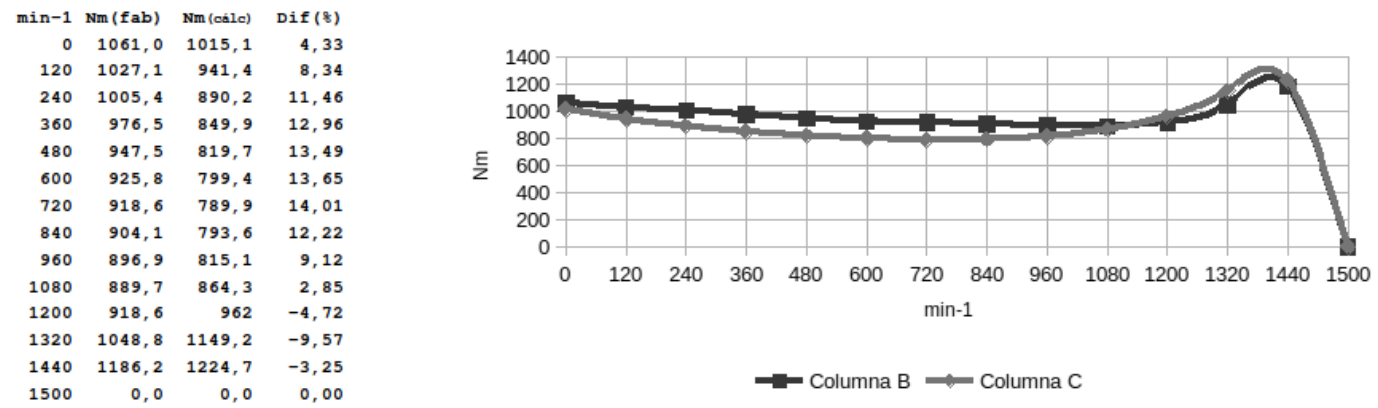

Fig. 2. Comparativa de curvas de momentos, $75 \mathrm{~kW}, 4$ polos 
La comparación de corrientes, para 75 kW, puede verse en la Fig. 3.

Referencias de la Fig. 3:

Columna A: velocidad, min-1

Columna B: Corriente según curva del fabricante ( $f a b), A$

Columna C: Corriente curva de cálculo (calc), $\mathrm{Nm}$

Columna D: Diferencia $100^{*}(B-C) / B$
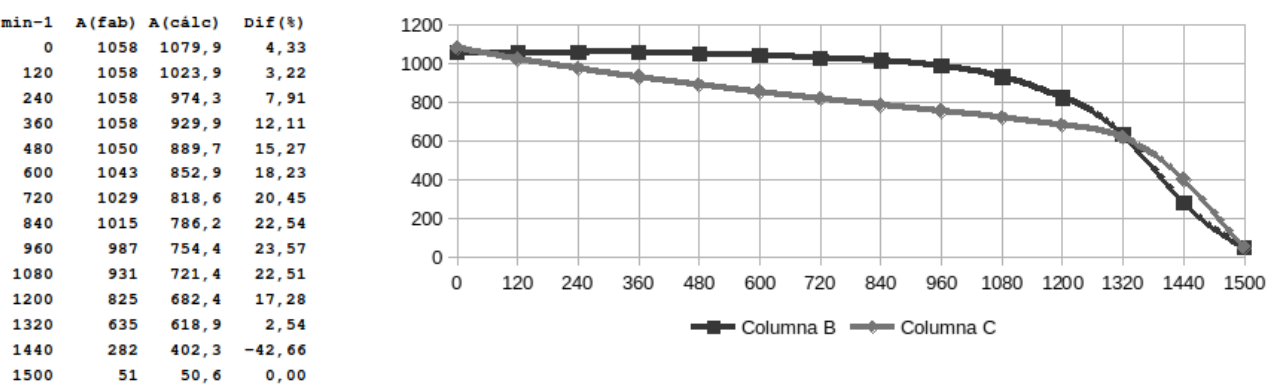

Fig. 3. Comparativa de curvas de corrientes, 75 kW, 4 polos

Los valores del fabricante fueron obtenidos de las gráficas otorgadas en la hoja de datos del motor.

En caso de comparación con curvas del fabricante de otros motores mencionados, los resultados se muestran en las figs 4 y 5 .

Referencias de las Figs 4 y 5 :

Columna B: valores del fabricante, $\mathrm{Nm} / \mathrm{A}$

Columna C: valores calculados, $\mathrm{Nm} / \mathrm{A}$
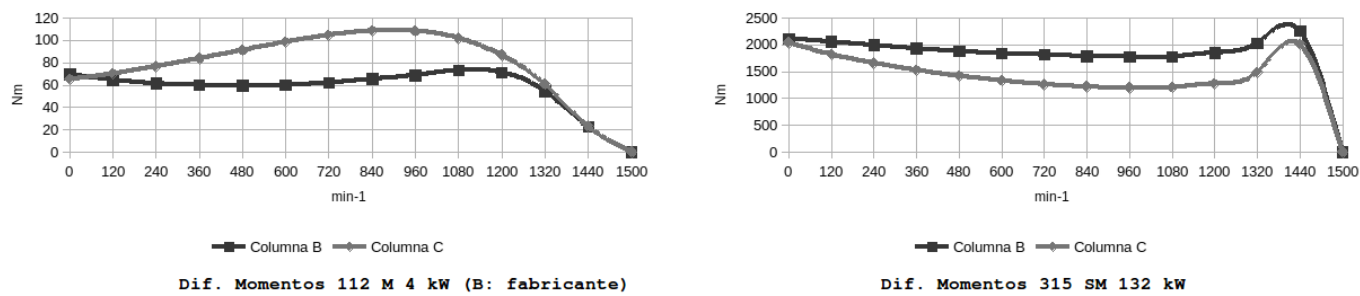

Fig 4. Comparativa de curvas de momentos de otros motores, 4 polos
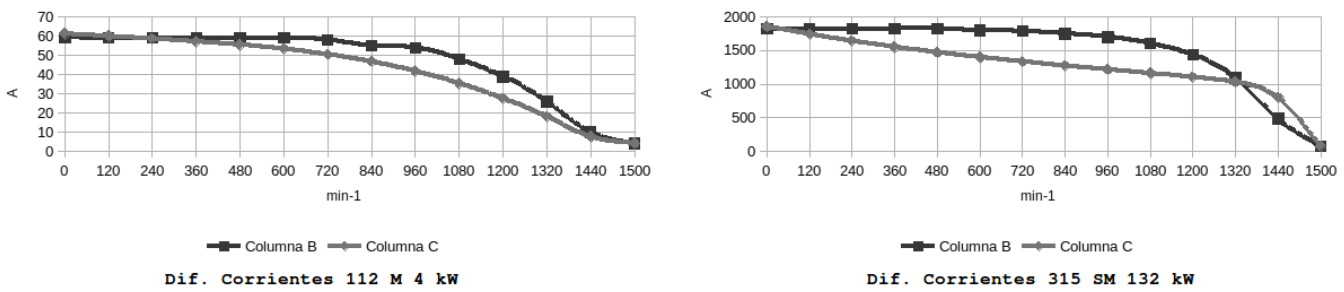

Fig 5. Comparativa de curvas de corrientes de otros motores, 4 polos 
A primera vista se puede observar en las figs. 2, 3, 4 y 5 que las diferencias en los valores se van incrementando hacia las zonas alejadas de los extremos de la curva (arranque y estados de carga, respectivamente); esto hace pensar que se requiere replantear, aparte de las pérdidas mecánicas y adicionales, la ley de variación de resistencia rotórica y reactancias, adaptándola a cada motor en particular. Como ya se anticipó en el punto d) del título anterior, ésto se puede lograr obteniendo mayor cantidad de muestras de impedancias en los puntos interiores de la curva del fabricante.

c) Graficación de curvas de las distintas magnitudes: Las curvas obtenidas de algunos de los parámetros, para $75 \mathrm{~kW}, 4 \mathrm{~kW}$ y $132 \mathrm{~kW}$ en 4 polos, se muestran en las figs. 6 a 11; sus formas obedecen a las que propone la bibliografía (Chapman, 2005: 401-430) y se acercan a las del fabricante.

Referencias sobre las figs 6 a 11 :

Columna B: potencia de entrada, $W /$ tensión de salida, $V /$ corriente primaria, $A$

Columna C: potencia en el eje (salida), $W /$ cdt rama derivada, $V /$ corriente secundaria, $A$

Columna D: corriente de la rama derivada, $A$
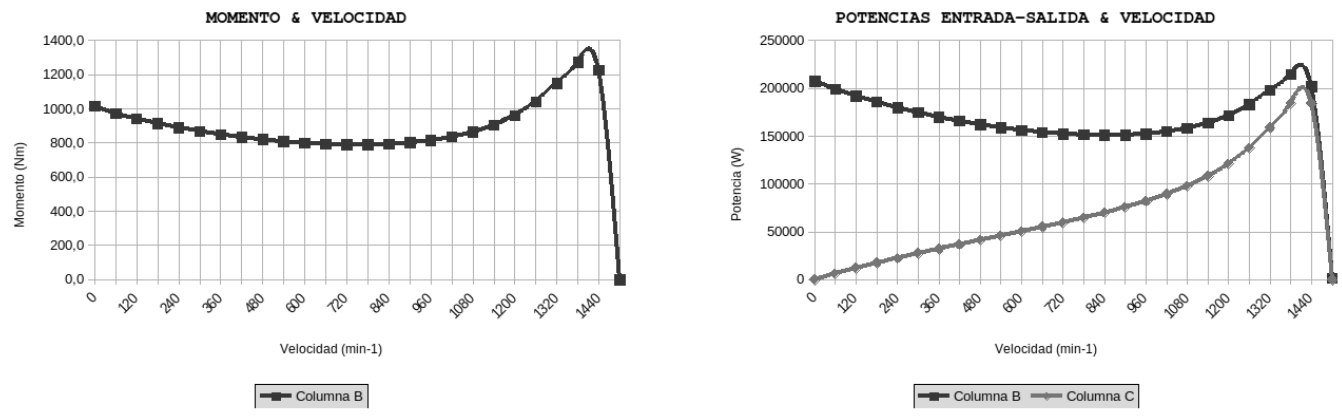

Fig. 6. Curvas de momentos y potencias, $75 \mathrm{~kW}, 4$ polos
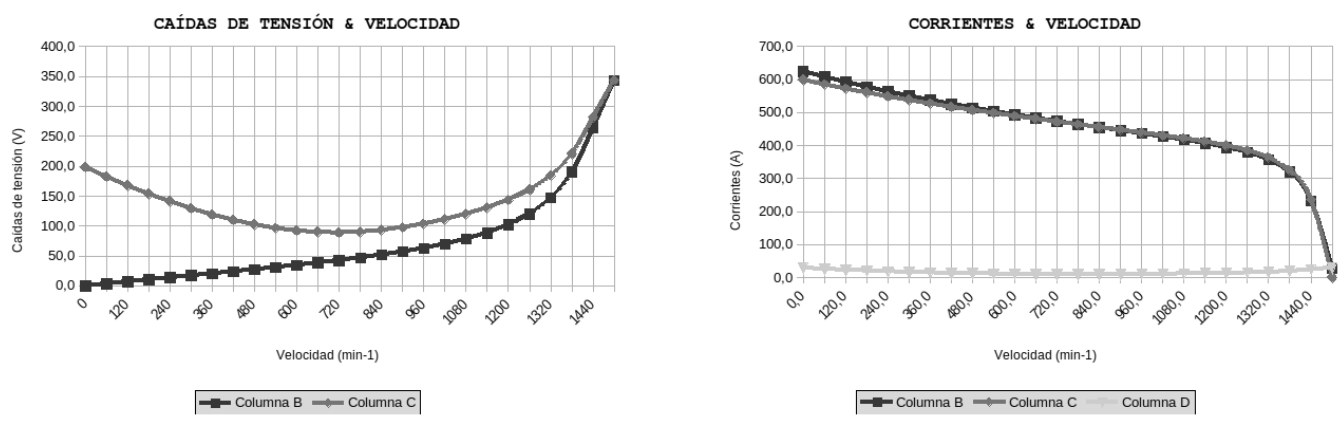

Fig. 7. Curvas de caídas de tensión y corrientes, 75 kW, 4 polos 

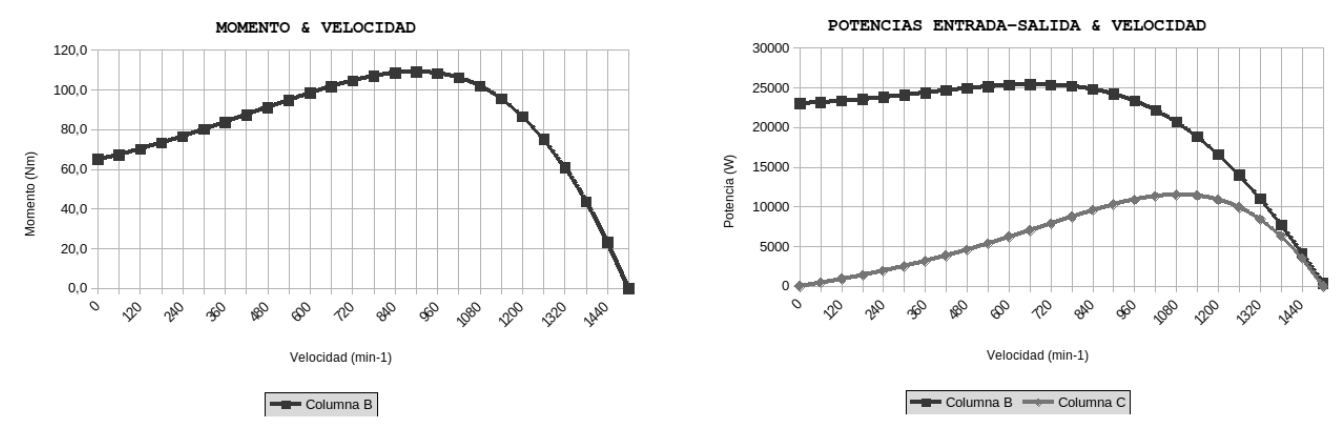

Fig. 8. Curvas de momentos y potencias, 4 kW, 4 polos
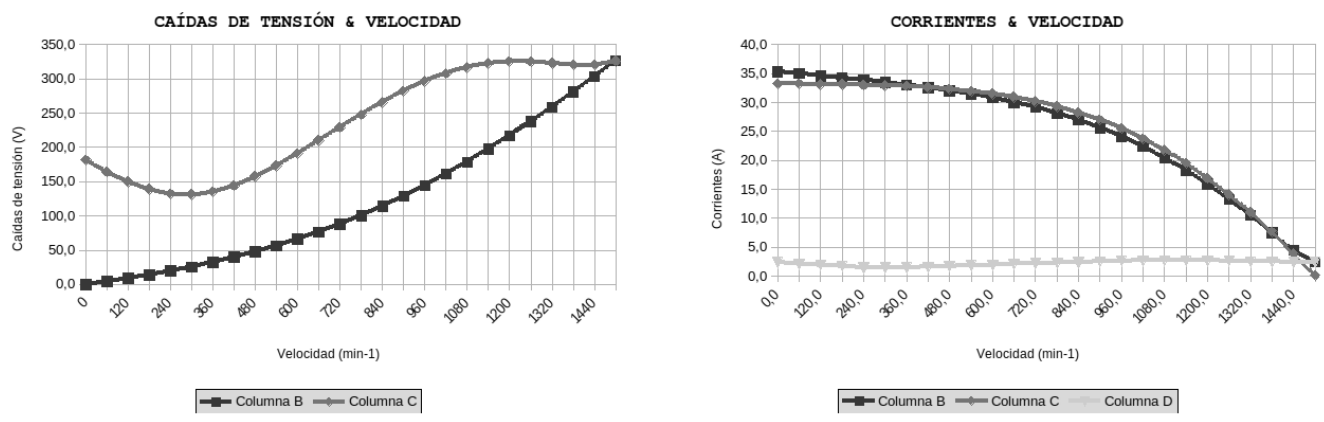

Fig. 9. Curvas de caídas de tensión y corrientes, 4 kW, 4 polos
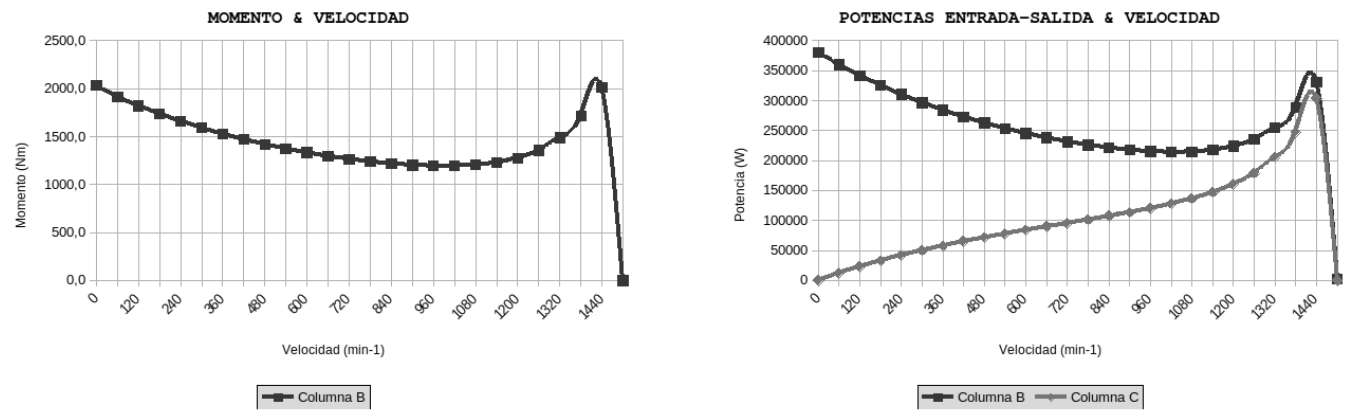

Fig. 10. Curvas de momentos y potencias, 132 kW, 4 polos 

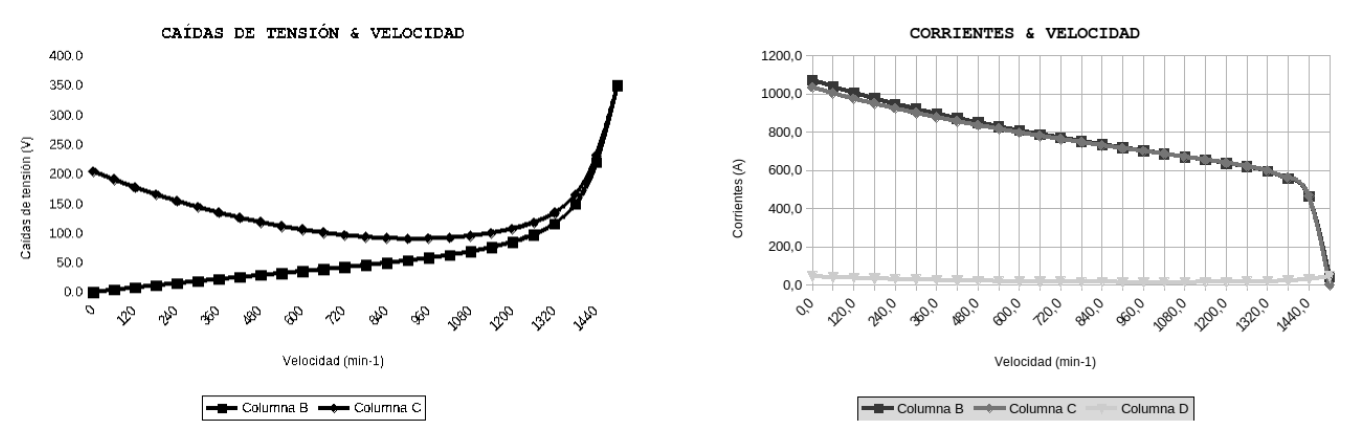

Fig. 11. Curvas de caídas de tensión y corrientes, 132 kW, 4 polos

Las curvas de momentos muestran claramente la diferencia entre los motores con rotor de doble jaula ( $75 \mathrm{~kW}$ y $132 \mathrm{~kW}$ ), es decir, alto momento de arranque, bajo deslizamiento y momento máximo a elevadas velocidades, con los de simple jaula (4 kW), tal como ilustra la bibliografía (Chapman, 2005: 417-424). Las formas de las curvas de potencias, caídas de tensión y corrientes también se muestran muy distintas cuando los rotores difieren en sus características; es de notar que en el caso de $4 \mathrm{~kW}$, en un tramo de la curva, el valor absoluto de la corriente secundaria supera al de la primaria, debido a valores negativos de X2 (aparente efecto capacitivo). Esto suele ocurrir frecuentemente en este modelo de cálculo.

d) Elaboración de tablas comparativas anexas: opcionalmente y como una extensión de las prestaciones del programa, resultaría de utilidad elaborar una tabla con datos constructivos (tabla 11) y una comparativa (tabla 12) de valores de exigencias para distintos tamaños y potencias de motores. Como ejemplo, se muestran las correspondientes a una linea comercial de motores de 4 polos.

Los datos de estas tablas son muy valiosos cuando se fabrica un prototipo de motor, ya que constituyen una fuente de información fundamental para acercar los valores calculados a los requeridos, ajustando las dimensiones. Tal método de aproximación es usado comúnmente por los diseñadores de máquinas.

\begin{tabular}{|c|c|c|c|c|c|c|c|c|c|c|c|c|c|c|c|}
\hline Altura del eje & $(\mathrm{mm})$ & 71 & 80 & 90 & 100 & 112 & 132 & 160 & 180 & 200 & 225 & 250 & 280 & 315 & 315 \\
\hline Potencias & $(\mathrm{kw})$ & 0,37 & 0,75 & 1,35 & 3,00 & 4,00 & 9,30 & 15,0 & 22,0 & 30,0 & 45,0 & 55,0 & 90,0 & 110 & 132 \\
\hline iámetro exterior estator & $(\mathrm{mm})$ & 120,5 & 120,5 & 130,5 & 145,5 & 165,3 & 195,3 & 240,3 & 260,3 & 295,0 & 330,3 & 430,0 & 430,5 & 495,0 & $495, c$ \\
\hline Altura corona estator & $(\mathrm{mm})$ & 11,8 & 11,8 & 11,8 & 12,9 & 15,4 & 18,9 & 24,5 & 21,7 & 26,5 & 32,7 & 47,5 & 47,8 & 52,5 & 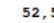 \\
\hline Altura corona rotor & $(\mathrm{mm})$ & 11,8 & 11,8 & 11,6 & 12,9 & 14 & 19,5 & 17 & 15,1 & 18,2 & 27,2 & 43,7 & 43,7 & 44,2 & 44,2 \\
\hline Diámetro eje rotor & $(\mathrm{mm})$ & 25 & 25 & 30 & 32 & 38 & 48 & 60 & 65 & 75 & 75 & 95 & 95 & 120 & $12 \mathrm{C}$ \\
\hline Longitud paquete de chapas & $(\mathrm{mm})$ & 45 & 80 & 115 & 136 & 115 & 210 & 235 & 260 & 250 & 280 & 200 & 330 & 340 & $39 c$ \\
\hline Número ranuras estator & - & 24 & 24 & 36 & 36 & 36 & 36 & 36 & 48 & 48 & 48 & 48 & 48 & 60 & 60 \\
\hline Espesor anillo rotor & $(\mathrm{mm})$ & 10 & 10 & 10 & 10 & 10 & 17,5 & 14 & 22 & 19 & 19 & 15 & 15 & 20 & 20 \\
\hline sección anillo rotor & $\left(\mathrm{mm}^{2}\right)$ & 128 & 128 & 134 & 151 & 170 & 289 & 392 & 713 & 698 & 709 & 657 & 657 & 1016 & 101 \\
\hline Longitud entrehierro & $(\mathrm{mm})$ & 0,35 & 0,35 & 0,30 & 0,30 & 0,25 & 0,35 & 0,40 & 0,60 & 0,50 & 0,60 & 0,80 & 0,80 & 1,00 & 1,0 \\
\hline
\end{tabular}

Tabla 11. Aspectos dimensionales de una línea de motores de 4 polos (anexo) 


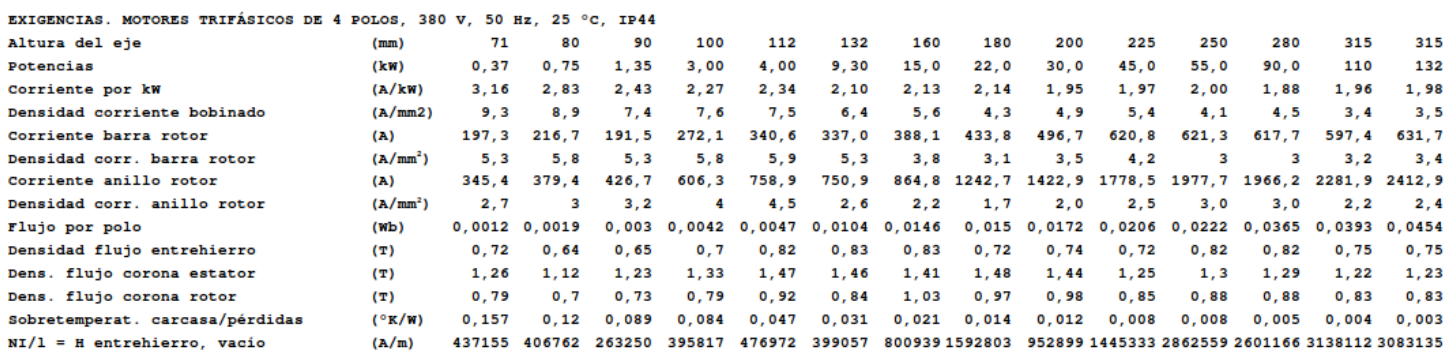

Tabla 12. Exigencias aproximadas de motores de una marca comercial (anexo)

Entre otras características de la tabla 12, se suelen tomar como referencias con el aumento de tamaño: a) la reducción de la densidad de corriente admisible, debido a la menor capacidad de disipación térmica de los conductores, b) el incremento del valor del flujo, que es proporcional a la potencia, c) la densidad de flujo en el entrehierro variando aproximadamente entre $0.6 \mathrm{~T}$ y $0.8 \mathrm{~T}$ y en la corona del estator, que no supera $1,5 \mathrm{~T}$, como valor razonable para el tipo de chapa usada, e) la reducción de la relación sobretemperatura carcasa/ pérdidas, debido al mayor volumen, superficie de disipación y capacidad de ventilación y f) el incremento de fuerza magnetomotriz en el entrehierro, por la mayor longitud de éste. Algunos de estos valores son diferentes en versiones con distinta protección, más modernas o complejas de motores.

\section{Conclusiones}

En el momento de redacción de este trabajo, se considera que la investigación aún se encuentra en sus etapas iniciales; no obstante, es admisible adelantar algunas conclusiones preliminares que en el futuro estarán sujetas a los nuevos análisis y resultados obtenidos durante la continuación de las experiencias.

a) Lenguaje de programación: luego de haber ejecutado el programa con múltiples tipos de motores, Python (González Duque, 2019) arrojó una respuesta acorde a las expectativas junto a una gran versatibilidad y estabilidad, permitiendo los sucesivos ajustes y ampliaciones necesarios para arribar a los requisitos fijados. Este modelo de software podría extenderse hacia la realización de otros trabajos, tales como el estudio de motores de dos velocidades, monofásicos o de imanes permanentes.

b) Curvas de momentos y corrientes, en función de la velocidad: las formas de las curvas se acercan a lo indicado por los estudios especializados y los catálogos; sin embargo existen, como ya se dijo, diferencias considerables con las gráficas del fabricante en la zona media de la curva. Esto conduce, además de necesitar un mayor número de comparaciones con dichas curvas del fabricante y analizar estadísticamente los resultados, a repensar la valoración de pérdidas y las leyes de variación de las impedancias, adaptándolas, muy probablemente, a cada tipo de potencia y velocidad de motor. En caso de prototipos de motores, en los que no existan curvas de referencia, será necesario obtener algunas estimaciones sobre las desviaciones de los valores teóricos calculados.

c) Cálculos aproximados de exigencias: ampliamente justificados por autores 
tradicionales reconocidos (Corrales Martín, 1976: 380-430; Crisci, 1956: 675-815) , estos valores se prestan a ser valiosos auxiliares de referencia para proyectos o diseños de máquinas. Las curvas de corrientes y de caídas de tensión reflejan sus variaciones con la velocidad en cada tramo del circuito, permitiendo aplicarlos a cálculos de máximos y mínimos en distintas zonas del motor.

d) Efectos transitorios y armónicos: No se consideran. Todos estos análisis son para el régimen permanente y para condiciones de cierta linealidad en la alimentación.

Finalmente, se puede decir que este software, concebido en un lenguaje accesible y moderno de programación y aplicando los principios tradicionales de análisis teórico y los datos de ensayos o constructivos del motor trifásico de inducción, es un recurso que puede proveer valores integrales de su funcionamiento, aplicables libremente en el ámbito académico, de diseño, servicio de reparación y producción. 


\section{Referencias}

Amaral, G.F.V. et al (2021) "A High Precision Method for Induction Machine Parameters Estimation From Manufacturer Data”. IEEE Transactions on. 36(2):1226-1233 Energy Conversion, USA. Disponible en: http://portal.bibliotecas.utn.edu.ar/proxy/https:// ieeexplore.ieee.org/document/9234616

Chapman, S. (2005). “Máquinas Eléctricas”. México: Mc Graw Hill, 401-430.

Crisci, G. (1956). "Costruzione, schemi e calcolo degli avvolgimenti delle macchine electtriche rotanti”. Módena: STFM Mucchi: 675-815. Disponible en: <https://www.mucchieditore. it/index.php?option=com_virtuemart\&view=productdetails\&virtuemart_product_ $\mathrm{id}=2168 \&$ virtuemart_category_id=67>

Corrales Martín, J. (1976). "Cálculo industrial de máquinas eléctricas”. Tomo I . Barcelona: Marcombo B.E.: 236-276.

Corrales Martín, J. (1976). “Cálculo industrial de máquinas eléctricas”. Tomo II. Barcelona: Marcombo B.E.: 380-430.

EASA/AEMT. (2003). "El Efecto de la Reparación/Rebobinado en la Eficiencia del Motor Estudio de Rebobinado EASA/AEMT y Guía de Buenas Prácticas Para Conservar La Eficiencia del Motor". USA, 18-19. Disponible en: <https://www.academia.edu/36573565/El_Efecto_de_la_ Reparaci\%C3\%B3n_Rebobinado_en_la_Eficiencia_del_Motor_Estudio_de_Rebobinado_ EASA_AEMT_y_Gu\%C3\%ADa_de_Buenas_Pr\%C3\%A1cticas_Para_Conservar_La_ Eficiencia_del_Motor>

Fraile Mora, J. (2008). “Máquinas Eléctricas”. Madrid: Mc Graw Hill, 259-334.

Gallo, O. (2017). "Convenio Universidad - Empresa. Grupo CIDEME. Servicios, investigación $y$ formación de estudiantes tecnológicos”. Editorial edUTecNe de la UTN. Disponible en: https://ria.utn.edu.ar/bitstream/handle/20.500.12272/3356/2017\%20CIDEME. pdf? sequence $=1 \&$ isAllowed $=\mathrm{y}$

Gallo, O. y Gallo, J. (2014). "Programa de Gestión de Bobinados (ProGeBo) (Primera parte)". Revista Iberoamericana de Ingeniería Industrial. Brasil, Vol. 6, $\mathrm{N}^{\circ} 11$, ISSN 21758018, pp. 328-348, noviembre 2014. Disponible en: https://ria.utn.edu.ar/handle/20.500.12272/3331

Gallo, O y Gallo, J. (2017). "Programa de Gestión de Bobinados (ProGeBo). Parte II". $46^{\circ}$ Congreso JAIIO, Córdoba, Argentina. Disponible en: https://ria.utn.edu.ar/ handle/20.500.12272/3359

González Duque, R. (2019). "Python para todos”. Disponible en: <http://mundogeek.net/ tutorial-python/>

Juha Pyrhönen, J. et al. , (2008). “Design of Rotating Electrical Machines”. UK: John Wiley \& Sons, Ltd. : 225-280.

LafertGroup (2021). "IE3 Range. Premium Efficiency Three Phase Motors". Italia. Disponible en: <https://www.lafertaust.com.au/wp-content/uploads/2019/01/Brochure-IE3-Range-2019. pdf $>$

Liwschitz-Garik, M; Whipple, C. (1974). "Máquinas de corriente alterna”. México: Editorial CECSA: 177-209. 
Norma IEC 60034-1. (2017). "Rotating electrical machines - Part 1: Rating and performance". Disponible en: <https://www.une.org/encuentra-tu-norma/busca-tu-norma/iec?c=28145>

Norma IEC 60034-2-1. (2014). Rotating electrical machines - Part 2-1: Standard methods for determining losses an efficiency from tests (excluding machines for tracción vehicles). Disponible en: <https://www.une.org/encuentra-tu-norma/busca-tu-norma/norma/?c=N0053468>

Python Software Foundation. (2021). "Documentation”. Disponible en: <https://www. python.org/>

Sale A, A. et al. (2018). "Optimization of Induction Motor Equivalent Circuit Parameter Estimation Based on Manufacturer's Data”. Energies, 2018, 11, 7, 1. Disponible en: $<$ http://portal.bibliotecas.utn.edu.ar/proxy/https://ideas.repec.org/a/gam/jeners/ v11y2018i7p1792-d156869.html>

WEG. (2021). "Motor Modular IE2 110 kW 4P 280S/M 3F 200/346/400/690//230/400 V 50 Hz IC411 - TEFC - B. Curva de par y corriente x rotación”. Brasil. Disponible en: $<$ https://www. weg.net/catalog/weg/ES/es/Motores-El\%C3\%A9ctricos/Motores-para-Aplicaci\%C3\%B3nIndustrial/Motor-Modular/Motor-Modular-\%28Hierro-Gris\%29/Motor-Modular-IE2-110-kW4P-280S-M-3F-200-346-400-690-230-400-V-50-Hz-IC411---TEFC---B3T/p/12411261>

Wengerkievicz C. et al. (2017). "Estimation of Three-Phase Induction Motor Equivalent Circuit Parameters from Manufacturer Catalog Data". Journal of Microwaves, Optoelectronics and Electromagnetic Applications. March 2017 16(1): 90-107. Disponible en: <http://portal. bibliotecas.utn.edu.ar/proxy/http://www.scielo.br/scielo.php?script=sci_arttext\&pid=S217910742017000100090\&lng=en\&tlng=en> 